\title{
First EVN Maps of 6-GHz OH Lines in Star Forming Regions
}

\section{J.-F. Desmurs ${ }^{1}$}

Observatoire de Bordeaux, BP 89, 33270 Floirac, France

JIVE / Observatorio Astronómico Nacional, 28800 Alcalá de Henares, Spain

\author{
A. Baudry \\ Observatoire de Bordeaux, BP 89, 33270 Floirac, France
}

\begin{abstract}
We have used 3 antennas of the EVN to observe in 6 star-forming regions simultaneously, and for the first time, the 2 main lines of the $J=\frac{5}{2}$ state of $O H$ with right and left circularly polarized feeds. Maser features and Zeeman pairs are identified by searching for emission over adjacent channels, and adjacent positions (within one synthesized beam) in both polarizations after we had mapped and used one selected simple channel as a reference. The magnetic field strength is thus estimated from the Zeeman pairs identified in our OH maps. We briefly present results obtained for $\mathrm{W} 3(\mathrm{OH}), \mathrm{ON} 1$, and $\mathrm{W} 51$.
\end{abstract}

\section{Introduction}

The $\mathrm{OH}$ radical is found in many different interstellar objects of our Galaxy and in outer galaxies. The $\mathrm{OH}$ level populations are easily inverted and this gives rise to powerful maser emissions. Moreover, the $\mathrm{OH}$ sensitivity to the magnetic field allows us to make accurate measurements of the field strength via the Zeeman effect. $\mathrm{OH}$ masers are prominent in compact $\mathrm{H}$ II regions, the birth sites of massive $\mathrm{O}$ or $\mathrm{B}$ stars. At this stage of the stellar evolution the magnetic field might play an important role. In the first excited state of $\mathrm{OH}$ the spectra are simpler than those of the ground state and we can rather easily interpret in terms of the Zeeman effect the eventual shift in frequency observed in right and left circularly polarized components. We present some results of the first EVN experiments made at $6 \mathrm{GHz}$. Our data will be analyzed more extensively elsewhere (Desmurs et al., in preparation). We briefly report here on results obtained in three massive star-forming regions (W3 $(\mathrm{OH}), \mathrm{ON} 1$ and $\mathrm{W} 51)$. We observed simultaneously the two main lines of the first excited state of $\mathrm{OH}\left({ }^{2} \Pi_{\frac{3}{2}}\right.$, $\mathrm{J}=\frac{5}{2}$ state) lying at $6035.092 \mathrm{MHz}$ and $6030.747 \mathrm{MHz}$ for the hyperfine transition $F: 3-3$ and $F: 2-2$, respectively. The study of this level is also important to give constraints on maser pumping models. VLBI observations are needed to search for the spatial coincidence of the two components of a Zeeman pair. We recall that a similar experiment has been done only once in the past (Moran et al. 1978) and has never been repeated since then, and we stress that the window around $6-7 \mathrm{GHz}$ is only available with the EVN.

\section{Observations}

In this first experiment performed in May 1994, we used three EVN antennas, Effelsberg, Jodrell Bank (Mk2) and Medicina. We recorded simultaneously the

\footnotetext{
${ }^{1}$ J.-F. D. wishes to acknowledge support for this research by the European Union under contract CHGECT 920011
} 
two main lines in both polarizations in MkIII mode $\mathrm{C}$ format. The data were correlated with the Bonn correlator, and were averaged over 10 seconds; the expected coherence time at this frequency is greater than 10 minutes. We reached a system temperature for Effelsberg of $60 \mathrm{~K}$ and of about $120 \mathrm{~K}$ for the two other antennas. The data reduction was made with the AIPS package developed by the NRAO. For each source, after a first calibration made from observations of a nearby calibrator, we have self-calibrated the data on a maser reference feature to correct for residual fringe rates. The reference feature in each source was chosen with the following criteria: 1) It shows a simple spatial structure. 2) It corresponds to a single maser source in the reference channel map. 3) It is strong enough to be easily detected. The solution thus obtained in one polarization was transferred to the other polarization. This allowed us to derive the relative positions of the maps in both polarizations as we first corrected all the instrumental effects causing a phase shift between the two polarizations. Finally, we produced maps of three sources in our sample. We searched for maser spots in each map deciding that a maser was detected when we were able to find emission at the same position in two or more contiguous channels with a peak intensity greater than 5 times the noise in the map. Then we searched for Zeeman pairs adopting the following conditions: 1) Two masers of opposite circular polarization are spatially coincident within one beam width. 2) The velocity separation between the two components is equal or greater than the maser line width.

\section{Results}

We have obtained, at the moment, $6 \mathrm{GHz} \mathrm{OH}$ maps for three of the six sources that we observed (W3(OH), ON1 and W51). All three sources were detected in the strongest main line ( $\mathrm{F}: 3-3$ at $6035 \mathrm{MHz})$ and only $\mathrm{W} 3(\mathrm{OH})$ was detected at $6031 \mathrm{MHz}$. We derived values of the magnetic field in each of this source. For $\mathrm{W} 3(\mathrm{OH})$, we mapped a region of 2 by 2 arc second with a resolution of 5 by 6.5 mas. We discovered 62 masers at $6035 \mathrm{MHz}$ in the two polarizations and identified 29 Zeeman pairs. At $6031 \mathrm{MHz}$ we discovered 23 masers and found 10 Zeeman pairs. The magnetic field strength lies in the range 1 to $10 \mathrm{mG}$ (Desmurs et al., in preparation). Our results confirm the earlier Zeeman measurements made at $6035 \mathrm{MHz}$ by Moran et al. (1978). Our new $6031 \mathrm{MHz}$ VLBI maps show a similar spatial distribution and give a value of the field consistent with our $6035 \mathrm{MHz}$ data (Desmurs et al., in preparation; Desmurs, Baudry, \& Graham 1997). ON1 was mapped with a resolution of 44 by 13 mas. We found 12 masers and we identified 2 Zeeman pairs giving a magnetic field between 1 and $3 \mathrm{mG}$. W51 was mapped with a resolution of 56 by 13 mas and showed 5 masers spot. Only one Zeeman pair and the field strength is around $3.5 \mathrm{mG}$.

\section{References}

Desmurs, J.-F., Baudry, A., \& Graham, D. 1997. Vistas in Astronomy, (special issue), eds. M. Garrett \& F. Colomer, 41, 169-184.

Moran, J. M., et al. 1978. ApJ, 224, L67-L71 